\title{
Can the 2019 AHA/ACC Cardiovascular Guidelines Be Improved?
}

\author{
David S. Schade ${ }^{1 *}$, Allen Adolphe ${ }^{2}$, Matthew Bouchonville ${ }^{3}$, R. Philip Eaton ${ }^{3}$ \\ ${ }^{1}$ Department of Internal Medicine/Endocrinology, University of New Mexico School of Medicine, Albuquerque, NM, USA \\ ${ }^{2}$ Division of Internal Medicine, Department of Internal Medicine, University of NM School of Medicine, Albuquerque, NM, \\ USA \\ ${ }^{3}$ Division of Endocrinology, Department of Internal Medicine, University of NM School of Medicine, Albuquerque, NM, USA \\ Email: ^dschade@salud.unm.edu, rpeaton@salud.unm.edu
}

How to cite this paper: Schade, D.S. Adolphe, A., Bouchonville, M. and Eaton, R.P. (2020) Can the 2019 AHA/ACC Cardiovascular Guidelines Be Improved? World Journal of Cardiovascular Diseases, 10, 72-77. https://doi.org/10.4236/wjcd.2020.102009

Received: December 23, 2019

Accepted: February 17, 2020

Published: February 20, 2020

Copyright (C) 2020 by author(s) and Scientific Research Publishing Inc. This work is licensed under the Creative Commons Attribution International License (CC BY 4.0).

http://creativecommons.org/licenses/by/4.0/

\begin{abstract}
The 2019 ACC/AHA Guideline on the Primary Prevention of Cardiovascular Disease for treatment of atherosclerosis in asymptomatic individuals is an advance over previously published recommendations. However, since all guidelines are based on a limited quantity of published studies and result from a consensus of experts in different fields of medicine, omissions and errors are inevitable. The present manuscript identifies four areas in these 2019 AHA/ACC guidelines that alternative approaches or changes would result in improved outcomes and reductions in atherosclerotic events. First, the goal for the reduction in the prevalence of cardiovascular disease should be total $(100 \%)$ eradication of the disease. This is a feasible goal as the facilities and resources to accomplish this task are currently available. Second, guidelines should acknowledge that atherosclerosis is a reversible disease as has previously been documented by multiple studies. If reversible, then under the appropriate clinical circumstances, it is preventable. Third, the goal for LDL cholesterol reduction should be $<50 \mathrm{mg} / \mathrm{dl}$, if eradication of atherosclerosis is to be achieved. This goal is achievable and safe as suggested by published studies. Fourth, widespread use of the coronary artery calcium scan needs to be recommended so that early atherosclerosis can be reversed before a major cardiovascular event occurs. Treating all individuals at a specific risk category without regard for the presence of disease results in poor adherence to therapy and unnecessary side effects. Consideration of these four issues would improve the AHA/ACC guidelines and result in better patient care.
\end{abstract}

\section{Keywords}

Guidelines, Diabetes, Risk/Benefit, LDL Cholesterol, LDL Goals 


\section{Introduction}

In 2019, the American Heart Association/American College of Cardiology issued their latest guidelines (2019 AHA/ACC guidelines) for treating asymptomatic individuals to prevent atherosclerotic cardiovascular disease (ASCVD) [1]. The 2019 AHA/ACC guideline is a resource for the clinical and public health practice communities. It addresses the primary prevention of atherosclerotic cardiovascular disease in adults, focuses on outcomes of acute coronary syndromes, myocardial infarction, stable or unstable angina, arterial revascularization, stroke, transient ischemic attack, or peripheral arterial disease of atherosclerotic origin, as well as heart failure and atrial fibrillation. The 2019 AHA/ACC guideline presents recommendations to prevent cardiovascular disease that are related to lifestyle factors (e.g., diet and exercise), other factors affecting cardiovascular disease risk (e.g., obesity, diabetes, blood cholesterol, hypertension, smoking, aspirin use), patient-centered approaches (e.g., team-based care, shared decision-making, assessment of social determinants of health), and considerations of the cost and value of primary prevention.

However, guidelines in general suffer from several deficiencies, including the choice of what data to review, the interpretation of scientific studies, and obtaining a consensus from a heterogeneous group of experts. For this reason, cardiovascular guidelines from professional organizations rarely agree [2]. This commentary raises four issues that provide an alternative approach to recent cardiovascular data that should result in the improvement of the 2019 AHA/ACC guidelines (Figure 1).

\section{The Goal for Reduction in Atherosclerosis Should Be $100 \%$}

The stated goal of the American Heart Association (AHA) for the reduction in

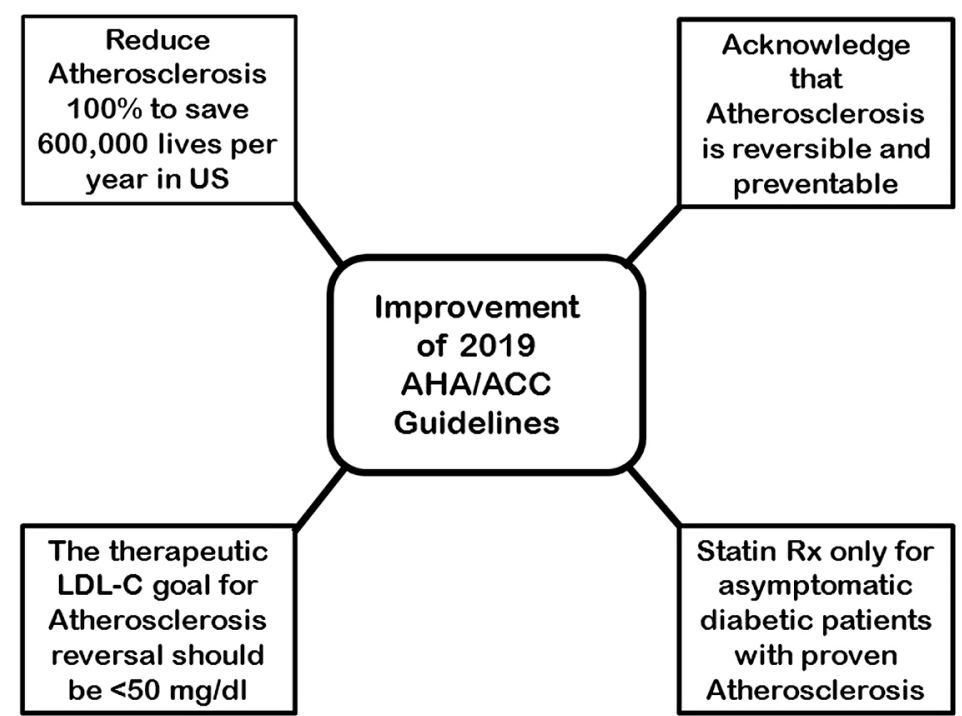

Figure 1. The four main changes that should be made to improve the 2019 AHA/ACC atherosclerosis guidelines. See text for details. 
atherosclerotic cardiovascular disease is $20 \%$ [3]. This goal is important because it determines the aggressiveness of the recommendations made in the guidelines. In the United States, approximately 600,000 people die of ASCVD each year [3]. Reducing this number by $20 \%$ would leave 480,000 deaths each year, the size of a small city. Since ASCVD is preventable and reversible in asymptomatic persons, a $20 \%$ reduction is untenable. The goal should be total elimination within a five-year period. What is required is the determination to achieve this goal. As recently detailed, the resources and technology are currently available to reach this goal [4].

The 2019 AHA/ACC guidelines recommend a very limited use for coronary artery calcium scanning (CAC) in order to aid therapeutic decisions in intermediate level risk patients [1]. This recommendation precludes the use of CAC in high and low risk individuals (the latter represent the majority of the population at risk) [5]. However, when CAC scoring is applied to groups of individuals in either of these risk categories, 50\% of individuals are reclassified to an alternative risk category [5]. Individuals with zero CAC score rarely need pharmacological therapy but do need a repeat CAC scan in five years because $25 \%$ will become positive within that interval [6]. CAC scanning provides a noninvasive approach to identifying almost all adults with subclinical ASCVD. It is reasonable to CAC scan all adults by the age of 50 years because $40 \%$ will have a positive score by that age [7]. The guidelines need to reassess their restricted position on utilizing CAC scanning in asymptomatic individuals.

Additional information and details of the approach to eliminate cardiovascular disease can be found in references [4] and [8]. Accomplishing this goal will require approximately five years and a commitment from healthcare providers, their institutions, and major organizations that support cardiovascular research and patient care. However, the resulting cost savings will be great as well as the millions of lives saved. This commitment has previously been done for other epidemics. Cardiovascular disease ranks highly among lethal, widespread epidemics.

\section{Atherosclerosis Is Reversible}

Numerous studies have demonstrated that atherosclerosis is a reversible disease when LDL cholesterol (LDL-C) is sufficiently reduced. Based on a meta-analysis of these studies, reversibility can first be observed in some individuals at an LDL-C of $78 \mathrm{mg} / \mathrm{dl}$ [9]. However, since atherosclerosis is reversible, it must be preventable under similar pathophysiological conditions. This concept is extremely important because it suggests that in asymptomatic individuals, aggressive lowering of LDL-C will eliminate this disease [10]. The guidelines need to address the reversibility of atherosclerosis and make recommendations for eliminating ASCVD. No discussion of reversibility is included in the guidelines and the targets given for LDL-C reduction are not sufficient to reverse atherosclerosis in many individuals as described below. 
Atherosclerosis reversibility can be observed when the LDL cholesterol is reduced to approximately $70 \mathrm{mg} / \mathrm{dl}$ for two years [4] [11]. However, at that level, approximately $50 \%$ of individuals do not experience a reversal of atherosclerotic coronary artery plaques [12]. Therefore, a lower level of LDL cholesterol of 50 $\mathrm{mg} / \mathrm{dl}$ is preferred which is both safe and effective [13]. Achieving this level of LDL cholesterol is feasible in almost all individuals as long as an appropriate low cholesterol diet, ezetimibe, and statin therapy are employed [4]. A meta-analysis of atherosclerosis reversibility includes 20 different studies all documenting that disease in the coronary arteries is a reversible metabolic process [9].

\section{The Goal for LDL-C Should Be $50 \mathrm{mg} / \mathrm{dl}$}

The advantage of specifying predetermined goals for any treatment are that both physicians and patients know whether the prescribed intervention is achieving the appropriate level of response that will improve patient health. Although reversal of atherosclerosis can be first observed at an LDL-C of $\sim 70 \mathrm{mg} / \mathrm{dl}$, only some patients experience reversal, whereas many individuals demonstrate progression [12]. In this study, an average LDL-C of $72 \mathrm{mg} / \mathrm{dl}$ resulted in reversal of atherosclerosis in 55\% of individuals and progression in $45 \%$. This observation is consistent with the level of LDL-C being a normal distribution. Given these data, an LDL-C concentration of $70 \mathrm{mg} / \mathrm{dl}$ should not be the recommended goal. A preferred goal of $50 \mathrm{mg} / \mathrm{dl}$ is not only safe, but achievable in almost all individuals with appropriate hypolipemic therapy [13].

The AHA/ACC guidelines recommend statins be used at either high-intensity (high dose) or intermediate-intensity (moderate dose) depending upon the ASCVD risk score of the individual patient. There is no acknowledgment in the guidelines that the side effects of statins are dose related, both for myalgias and diabetes (the only two proven side effects of statins) [14] [15]. Since the reduction of ASCVD is proportional to the reduction in LDL-C, the current approach should be "the lower the better" as long as side effects of statins don't limit their use [16]. The guidelines do not acknowledge Robert's rule that doubling the dose of a statin only reduces LDL-C by $5 \%$ to $7 \%$ [17]. Therefore, physicians need to use the lowest dose of statins possible and still achieve significant reductions in LDL-C. The use of $10 \mathrm{mg}$ of ezetimibe in concert with $10 \mathrm{mg}$ of rosuvastatin will achieve significant reduction without an increase in statin side effects [4]. The guidelines should discuss this therapeutic regimen so that high dosages of statins are rarely needed.

\section{Not All Patients with Diabetes Should Be Prescribed Statins}

The 2019 AHA/ACC guidelines recommend that all asymptomatic patients with diabetes receive statin therapy irrespective of whether they have demonstrated atherosclerosis. This approach results in many patients with diabetes taking statins who do not have and will not develop atherosclerosis. As a result, poor 
compliance and unnecessary side effects are observed [18]. Because of major improvements in diabetes therapy and advances in technology, many diabetic patients achieve normal blood pressures, HbA1c, and blood lipids [19]. For these individuals, their risk of atherosclerosis with a zero calcium scan is no greater than the risk for individuals without diabetes [20]. Statin therapy may be inappropriate for these individuals. The guidelines need to acknowledge that "statins for all diabetes" is no longer a scientifically based position.

\section{Summary}

The 2019 AHA/ACC guidelines represent the latest consensus from organizations that attempt to improve the lives of individuals which are at risk for cardiovascular disease. As such, they include many good suggestions on the risk and prevention of atherosclerotic disease. However, because they require a consensus, several forward-looking issues requiring a change in thought processing may be overlooked. In this article, we have addressed four of these issues that are critical to preventing over treatment and under diagnosis of cardiovascular disease. We have offered four potential changes that would transform the 2019 AHA/ACC guidelines into a more robust document and result in the savings of thousands of lives.

\section{Conflicts of Interest}

The authors declare no conflicts of interest regarding the publication of this paper.

\section{References}

[1] Arnett, D.K., Blumenthal, R.S., Albert, M.A., et al. (2019) 2019 ACC/AHA Guideline on the Primary Prevention of Cardiovascular Disease. Circulation, 140, e596-e646.

[2] Khanji, M.Y., Bicalho, V.S., van Waardhuizen, C.N., Ferket, B.S., Petersen, S.E. and Hunink, M. (2016) Cardiovascular Risk Assessment: A Systematic Review of Guidelines. Annals of Internal Medicine, 165, 713-722. https://doi.org/10.7326/M16-1110

[3] Benjamin, E.J., Virani, S.S., Callaway, C.W., et al. (2018) Heart Disease and Stroke Statistics-2018 Update: A Report from the American Heart Association. Circulation, 137, e67-e492. https://doi.org/10.1161/CIR.0000000000000558

[4] Schade, D.S. and Eaton, R.P. (2019) A Simplified Approach to Reducing Cardiovascular Risk. The Journal of Clinical Endocrinology \& Metabolism, 104, 6033-6039. https://doi.org/10.1210/jc.2018-02509

[5] Silverman, M.G., Blaha, M.J., Krumholz, H.M., et al. (2014) Impact of Coronary Artery Calcium on Coronary Heart Disease Events in Individuals at the Extremes of Traditional Risk Factor Burden. European Heart Journal, 35, 2232-2241. https://doi.org/10.1093/eurheartj/eht508

[6] Min, J.K., Lin, F.Y., Gidseg, D.S., et al. (2010) Determinants of Coronary Calcium Conversion among Patients with a Normal Coronary Calcium Scan. Journal of the American College of Cardiology, 55, 1110-1117. https://doi.org/10.1016/j.jacc.2009.08.088

[7] Schade, D.S., Ramo, B., Obenshain, S.S., Schrader, R. and Eaton, R.P. (2019) The Rationale for a Different Approach to Preventing Cardiovascular Disease. World Journal of Cardiovascular Diseases, 9, 489-510. 
[8] Schade, D.S., Obenshain, S.S., Ramo, B. and Eaton, R.P. (2019) A Feasible, Simple, Cost-Saving Program to End Cardiovascular Disease in the United States. The American Journal of Medicine, 132, 1365-1367. https://www.amjmed.com/article/S0002-9343(19)30328-6/pdf

[9] Gao, W.Q., Feng, Q.Z., Li, Y.F., et al. (2014) Systematic Study of the Effects of Lowering Low-Density Lipoprotein-Cholesterol on Regression of Coronary Atherosclerotic Plaques Using Intravascular Ultrasound. BMC Cardiovascular Disorders, 14, 60-78. https://doi.org/10.1186/1471-2261-14-60

[10] Burge, M.R., Eaton, R.P., Comerci, G., Cavanaugh, B., Ramo, B. and Schade, D.S. (2017) Management of Asymptomatic Patients with Positive Coronary Artery Calcium Scans. Journal of the Endocrine Society, 1, 588-599.

https://doi.org/10.1210/js.2016-1080

[11] Nissen, S.E., Nicholls, S.J., Sipahi, I., Libby, P., Raichlen, J.S., Ballantyne, C.M., Davignon, J., Erbel, R., Fruchart, J.C., Tardif, J.-C., Schoenhagen, P., Crowe, T., Cain, V., Wolski, K., Goormastic, M. and Tuzcu, E.M. (2006) Effect of Very High-Intensity Statin Therapy on Regression of Coronary Atherosclerosis-The ASTEROID Trial. JAMA, 295, 1556-1565. https://doi.org/10.1001/jama.295.13.jpc60002

[12] Howard, B.V., Roman, M.J., Devereux, R.B., et al. (2008) Effect of Lower Targets for Blood Pressure and LDL Cholesterol on Atherosclerosis in Diabetes. The SANDS Randomized Trial. JAMA, 299, 1678-1689. https://doi.org/10.1001/jama.299.14.1678

[13] O’Keefe, J.H., Cordain, L., Harris, W.H., Moe, R.M. and Vogel, R. (2004) Optimal Low-Density Lipoprotein Is 50 to $70 \mathrm{mg} / \mathrm{dl}$ : Lower Is Better and Physiologically Normal. Journal of the American College of Cardiology, 43, 2142-2146. https://doi.org/10.1016/j.jacc.2004.03.046

[14] Collins, R., Reith, C., Emberson, J., et al. (2016) Interpretation of the Evidence for the Efficacy and Safety of Statin Therapy. The Lancet, 388, 2532-2561. https://doi.org/10.1016/S0140-6736(16)31357-5

[15] Schade, D.S., Shey, L. and Eaton, R.P. (2019) Prescribing Statins to Reduce Cardiovascular Disease: 10 Common Misconceptions. The American Journal of Medicine, 132, 897-899. https://doi.org/10.1016/j.amjmed.2019.01.042

[16] Schade, D.S., Cavanaugh, B., Ramo, B. and Eaton, R.P. (2016) The Application of the LDL Principle. World Journal of Cardiovascular Diseases, 6, 109-125. https://doi.org/10.4236/wjcd.2016.65012

[17] Roberts, W.C. (1997) The Rule of 5 and the Rule of 7 in Lipid-Lowering by Statin Drugs. American Journal of Cardiology, 80, 106-107. https://doi.org/10.1016/S0002-9149(97)00298-1

[18] Gomez Sandoval, Y.H., Braganza, M.V. and Daskalopoulou, S.S. (2011) Statin Discontinuation in High-Risk Patients: A Systematic Review of the Evidence. Current Pharmaceutical Design, 17, 3669-3689. https://doi.org/10.2174/138161211798220891

[19] Rawshani, A., Franzén, S. and Sattar, N. (2018) Risk Factors, Mortality, and Cardiovascular Outcomes in Patients with Type 2 Diabetes. The New England Journal of Medicine, 379, 633-644. https://doi.org/10.1056/NEJMoa1800256

[20] Agarwal, S., Cox, A.J., Herrington, D.M., et al. (2013) Coronary Calcium Score Predicts Cardiovascular Mortality in Diabetes: Diabetes Heart Study. Diabetes Care, 36, 972-977. https://doi.org/10.2337/dc12-1548 